\title{
Exact Potts/Tutte Polynomials for Polygon Chain Graphs
}

\author{
Robert Shrock ${ }^{1}$ \\ 1 C. N. Yang Institute for Theoretical Physics, Stony Brook University, Stony \\ Brook, NY 11794 \\ E-mail: robert.shrock@stonybrook.edu
}

\begin{abstract}
.
We present exact calculations of Potts model partition functions and the equivalent Tutte polynomials for polygon chain graphs with open and cyclic boundary conditions. Special cases of the results that yield flow and reliability polynomials are discussed. We also analyze special cases of the Tutte polynomials that determine various quantities of graph-theoretic interest.
\end{abstract}

PACS numbers: 05.50+q, 64.60.Cn, 68.35.Rh, 75.10.H 


\section{Introduction}

In Ref. [1] with S.-H. Tsai, exact results were given for the partition function of the zerotemperature $q$-state Potts antiferromagnet, or equivalently, the chromatic polynomial, on open and cyclic chain graphs composed of $m$ repetitions of $p$-sided polygons connected to each other by line segments. In this paper we generalize this work and give the full Potts model partition function for these families of graphs. The $q$-state Potts model has long been of interest in the study of phase transitions and critical phenomena [2. On a lattice, or, more generally, on a graph $G$, at temperature $T=1 /\left(k_{B} \beta\right)$, the partition function for this model is $Z=\sum_{\left\{\sigma_{i}\right\}} e^{-\beta \mathcal{H}}$, with the Hamiltonian $\mathcal{H}=-J \sum_{e_{i j}} \delta_{\sigma_{i} \sigma_{j}}$, where $J$ is the spin-spin interaction constant, $i$ and $j$ denote vertices on $G, e_{i j}$ is the edge connecting them, and $\sigma_{i}$ are classical spins taking on values in the set $\{1, \ldots, q\}$. We use the notation $K=\beta J$ and $v=e^{K}-1$. Thus, for the Potts ferromagnet $(J>0)$ and antiferromagnet $(J<0)$, the physical ranges of $v$ are $v \geq 0$ and $-1 \leq v \leq 0$, respectively. For the Potts antiferromagnet (PAF), $J<0$ so that, as $T \rightarrow 0, K \rightarrow-\infty$; hence, in this limit, the only contributions to the PAF partition function are from spin configurations in which adjacent spins have different values. The resultant $T=0$ PAF partition function is therefore precisely the chromatic polynomial $P(G, q)$ of the graph $G$, which counts the number of ways of assigning $q$ colors to the vertices of $G$ subject to the condition that no two adjacent vertices have the same color. (These are called proper $q$-colorings of $G$.)

In general, a graph $G=(V, E)$ is defined by its set of vertices (sites), $V$, and its set of edges (bonds), $E$. We denote the number of vertices of $G$ as $n=n(G)=|V|$ and the number of edges of $G$ as $e(G)=|E|$. The families of graphs to be considered here are open and cyclic chains of polygons connected by line segments. One may regard the chain as being oriented so that the longitudinal direction is horizontal. Each polygon is connected to the chain at two vertices, such that there are $e_{1}$ edges of the polygon above the chain and $e_{2}$ edges below the chain, and there are $e_{g}$ edges between each polygon (where $g$ stands for gap). Some illustrative examples are given in Fig. 11 (from [1]). The basic subgraph unit of the chain is thus a polygon with

$$
p=e_{1}+e_{2}
$$

edges, connected to a line segment with $e_{g}$ edges. The full chain with open (o) or cyclic (c) boundary conditions (BC) is comprised of $m$ repetitions of this basic subgraph comprised of the $p$-gon and $e_{g}$-length line segment, and is denoted, as in [1], by $G_{e_{1}, e_{2}, e_{g}, m ; B C}$. Since the two sides of the chain are equivalent, the interchange $e_{1} \leftrightarrow e_{2}$ leaves it invariant, so $G_{e_{1}, e_{2}, e_{g}, m ; B C}=G_{e_{2}, e_{1}, e_{g}, m ; B C}$. This implies that all of the quantities to be presented below are also invariant under this interchange. Indeed, in some of these quantities, the numbers $e_{1}$ and $e_{2}$ only enter in the form of their sum, $p$. Since the $(m+1)$ 'th member of a family of strip graphs of this sort can be obtained from the $m$ 'th member by gluing on an additional basic subgraph unit or, in the case of the cyclic strip, by cutting the strip transversely, inserting an additional basic subgraph unit 
and regluing, these are recursive families, in the sense of [3]. The numbers of vertices and edges of these graphs are

$$
\begin{aligned}
& n\left(G_{e_{1}, e_{2}, e_{g}, m ; o}\right)=\left(p+e_{g}-1\right) m+1, \\
& n\left(G_{e_{1}, e_{2}, e_{g}, m ; c}\right)=\left(p+e_{g}-1\right) m,
\end{aligned}
$$

and

$$
e\left(G_{e_{1}, e_{2}, e_{g}, m ; o}\right)=e\left(G_{e_{1}, e_{2}, e_{g}, m ; c}\right)=\left(p+e_{g}\right) m \text {. }
$$

Clearly, $G_{e_{1}, e_{2}, e_{g}, m ; o}$ and $G_{e_{1}, e_{2}, e_{g}, m ; c}$ are planar graphs. In the context of statistical mechanics, one takes $e_{1} \geq 1$ and $e_{2} \geq 1$, whence $p \geq 2$, since setting either $e_{1}=0$ or $e_{2}=0$ would mean that a spin $\sigma_{i}$ would interact with itself rather than with neighboring spins. However, in the context of mathematical graph theory, one may formally consider the case where $e_{1}=0, e_{2}=1$ or $e_{1}=1, e_{2}=0$, whence, $p=1$. In these cases, the graphs $G_{e_{1}, e_{2}, e_{g}, m ; B C}$ contain loops, where a loop is defined as an edge that connects a vertex back to itself.

One motivation for the present work is to understand how the results of [1] can be generalized to finite temperature and ferromagnetic as well as antiferromagnetic spinspin couplings. Another is to get further insight into how properties of a graph affect the Potts partition function $Z$ or equivalent nTutte polynomial (see further below). A particular appeal of the chain graphs considered here is that the results are sufficiently simple that one can study them in considerable explicit detail. For recursive strip graphs of length $m$ basic subunits, $Z$ is a sum of $m$ 'th powers of certain algebraic functions, generically denoted as $\lambda$ 's. Although calculations of $Z$ have been done on wider strips of regular lattices, as the strip width increases, the results rapidly become quite complicated. For example, for the cyclic (or Möbius) strip graph of the square lattice of width $L_{y}=3$ and length $L_{x}=m$, there are $\left(\begin{array}{l}6 \\ 3\end{array}\right)=20$ different $\lambda$ 's, and many of these are solutions of algebraic equations of sufficiently high degree so that they cannot be expressed in closed analytic form [5]. Although for a given width, fewer $\lambda$ 's occur for an open strip than for a cyclic strip, even for width $L_{y}=3$, there are five of these, including four that are solutions of a 4'th order algebraic equation, rendering an explicit expression rather cumbersome [5, 6]. Thus, it is valuable to investigate the effects of graphical properties on $Z$ for families of strip graphs where one can obtain explicit exact closed-form analytic solutions for the $\lambda$ 's that enter.

\section{General Background}

In this section we discuss some general background material relevant to our study. Let $G^{\prime}=\left(V, E^{\prime}\right)$ be a spanning subgraph of $G$, i.e. a subgraph having the same vertex set $V$ and an edge set $E^{\prime} \subseteq E$. Then $Z$ can be written as [7]

$$
Z(G, q, v)=\sum_{G^{\prime} \subseteq G} q^{k\left(G^{\prime}\right)} v^{e\left(G^{\prime}\right)},
$$


(a)

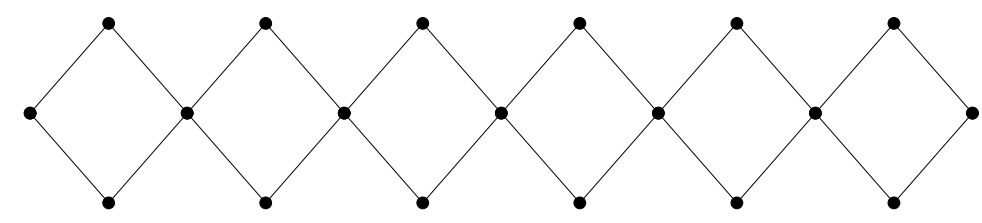

(b)

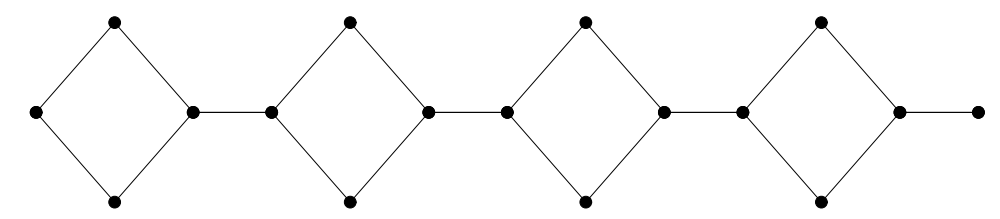

(c)

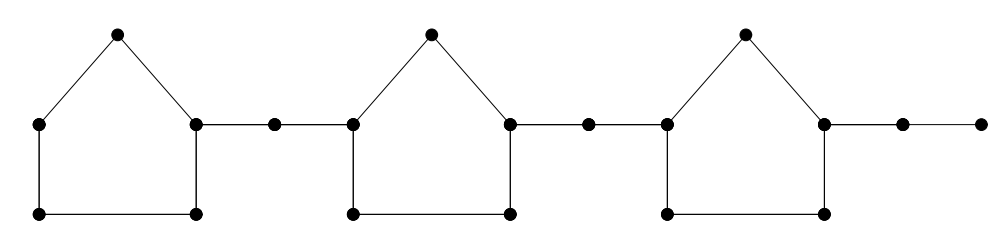

Figure 1. Illustrations of cyclic and open polygon chain graphs $G_{e_{1}, e_{2}, e_{g}, m}$ and $G_{e_{1}, e_{2}, e_{g}, m ; o}$ with $\left(e_{1}, e_{2}, e_{g}, m\right)=($ a) $(2,2,0,6)$, (b) $(2,2,1,4)$, (c) $(2,3,2,3)$. For the cyclic (open) chain graphs, the rightmost vertex on each graph is identified with (distinct from) the leftmost vertex at the same level, respectively.

where $k\left(G^{\prime}\right)$ denotes the number of connected components of $G^{\prime}$. As is evident from (2.1), $Z(G, q, v)$ is a polynomial in $q$ and $v$. In the ferromagnetic case with $v>0$, Eq. (2.1) allows one to extend the definition of $q$ from the positive integers to the positive real numbers while maintaining $Z(G, q, v)>0$ and hence a Gibbs measure. Since $k\left(G^{\prime}\right) \geq 1 \forall G^{\prime}$, it follows that $Z(G, q, v)$ always has a factor of $q$. It is thus convenient to define the reduced partition function $Z_{r}(G, q, v)$ as

$$
Z(G, q, v)=q Z_{r}(G, q, v)
$$

For a graph $G$, let us denote $G-e$ as the graph obtained by deleting the edge $e$ and $G$ /e as the graph obtained by deleting the edge $e$ and identifying the two vertices that were 
connected by this edge of $G$. This operation is called a contraction of $G$ on $e$. From Eq. (2.1), it follows that $Z(G, q, v)$ satisfies the deletion-contraction relation

$$
Z(G, q, v)=Z(G-e, q, v)+v Z(G / e, q, v) .
$$

The Potts model partition function is equivalent to the Tutte polynomial, $T(G, x, y)$, an object of considerable interest in mathematical graph theory. For a graph $G[8,9]$,

$$
T(G, x, y)=\sum_{G^{\prime} \subseteq G}(x-1)^{k\left(G^{\prime}\right)-k(G)}(y-1)^{c\left(G^{\prime}\right)},
$$

where $c\left(G^{\prime}\right)$ denotes the number of (linearly independent) cycles in $G^{\prime}$. Note that $c(G)=e(G)+k(G)-n(G)$. As is clear from (2.4), $T(G, x, y)$ is a polynomial in $x$ and $y$. All of the families of graphs considered here are connected, so that $k(G)=1$. Let us define

$$
x=1+\frac{q}{v}, \quad y=v+1,
$$

so that $q=(x-1)(y-1)$. Then the equivalence between $Z(G, q, v)$ and $T(G, x, y)$ is given by

$$
Z(G, q, v)=(x-1)^{k(G)}(y-1)^{n(G)} T(G, x, y) .
$$

The special case $v=-1$ defines the $T=0$ Potts antiferromagnet, and sets the variables in the Tutte polynomial equal to $x=1-q$ and $y=0$; in this case these functions yield the chromatic polynomial:

$$
Z(G, q,-1)=P(G, q)=(-q)^{k(G)}(-1)^{n(G)} T(G, 1-q, 0) .
$$

If $G$ contains a loop, then $T(G, x, y)$ contains a factor of $y$ and $P(G, q)$ vanishes identically, since it is not possible to satisfy the proper $q$-coloring condition.

We recall two elementary results. For any tree graph $T_{n}$ with $n$ vertices,

$$
T\left(T_{n}, x, y\right)=x^{n-1}, \quad Z\left(T_{n}, q, v\right)=q(q+v)^{n-1} .
$$

For the circuit graph with $n$ vertices, $C_{n}$,

$$
T\left(C_{n}, x, y\right)=\frac{x^{n}+c_{1}}{x-1}, \quad Z\left(C_{n}, q, v\right)=(q+v)^{n}+(q-1) v^{n} .
$$

where $c_{1}=x y-x-y=q-1$. Note that although $T\left(C_{n}, x, y\right)$ is expressed in Eq. (2.9) as a rational function, it is actually a polynomial, as is guaranteed by Eq. (2.4). Explicitly, $T\left(C_{1}, x, y\right)=y$ and, for $n \geq 2, T\left(C_{n}, x, y\right)=y+\sum_{j=1}^{n-1} x^{j}$. A similar comment applies to expressions below involving $(x-1)$ denominators. For the special case $v=-1$, since $P\left(C_{n}, q\right)$ has a factor $q(q-1)$ for $n \geq 2$, it is convenient to define the polynomial $D_{n}$ via

$$
D_{n}=\frac{P\left(C_{n}, q\right)}{q(q-1)}=\sum_{s=0}^{n-2}(-1)^{s}\left(\begin{array}{c}
n-1 \\
s
\end{array}\right) q^{n-2-s} .
$$

(For $n=1, P\left(C_{1}, q\right)=0$, since $C_{1}$ is a vertex with a loop; hence also $D_{1}=0$.) 


\section{Calculations and Results}

Using a systematic application of the deletion-contraction theorem, we have calculated the Potts partition function and equivalent Tutte polynomial for the open and cyclic chain graphs $G_{e_{1}, e_{2}, e_{g}, m ; o}$ and $G_{e_{1}, e_{2}, e_{g}, m ; c}$. For the open chain graph $G_{e_{1}, e_{2}, e_{g}, m ; o}$ we find, for the Tutte polynomial,

$$
T\left(G_{e_{1}, e_{2}, e_{g}, m ; o}, x, y\right)=\left(\lambda_{T, 0}\right)^{m},
$$

where

$$
\lambda_{T, 0}=T\left(T_{e_{g}+1}, x, y\right) T\left(C_{p}, x, y\right)=x^{e_{g}}\left(\frac{x^{p}+c_{1}}{x-1}\right) .
$$

Note that $\lambda_{T, 0}$ only depends on $e_{1}$ and $e_{2}$ via their sum, $p$. In general, if a graph $G$ can be expressed as $G_{1} \cup G_{2}$ such that $G_{1} \cap G_{2}$ is a single vertex, then $T(G, x, y)=$ $T\left(G_{1}, x, y\right) T\left(G_{2}, x, y\right)$. This factorization property holds for the basic subgraph units of $G_{e_{1}, e_{2}, e_{g}, m ; o}$, since the $p$-gon intersects the line segment at a single vertex. The factorization property also holds, a fortiori, for the open chain $G_{e_{1}, e_{2}, e_{g}, m ; o}$ itself. These facts imply the properties that (i) $\lambda_{T, 0}$ has the form of a product of the Tutte polynomials of the tree graph $T_{e_{g}+1}$ and of the circuit graph, $C_{p}$, and (ii) $T\left(G_{e_{1}, e_{2}, e_{g}, m ; o}, x, y\right)$ has the form of a power of a single polynomial, as given in Eq. (3.1). Corresponding comments apply to $Z\left(G_{e_{1}, e_{2}, e_{g}, m ; o}, q, v\right)$.

For the cyclic chain graph $G_{e_{1}, e_{2}, e_{g}, m ; c}$ we calculate

$$
T\left(G_{e_{1}, e_{2}, e_{g}, m ; c}, x, y\right)=\frac{1}{x-1}\left[\left(\lambda_{T, 0}\right)^{m}+c_{1}\left(\lambda_{T, 1}\right)^{m}\right],
$$

where $\lambda_{T, 0}$ and $c_{1}$ were given above and

$$
\lambda_{T, 1}=T\left(C_{e_{1}}, x, y\right)+T\left(C_{e_{2}}, x, y\right)+1-y .
$$

It is noteworthy that $\lambda_{T, 1}$ is independent of $e_{g}$ and only depends on the properties of the polygons, as encoded in their edge numbers $e_{1}$ and $e_{2}$.

From these general results, one can consider special cases of the various graphical edge numbers $e_{1}, e_{2}$, and $e_{g}$. For example, for the open and cyclic chain graphs $G_{2,2, e_{g}, m ; o}$ and $G_{2,2, e_{g}, m ; c}$, illustrated (for $e_{g}=0,1$ and $m=6,4$ ) in Fig. 1, we have

$$
T\left(G_{2,2, e_{g}, m ; o}, x, y\right)=\left[x^{e_{g}}\left(x+x^{2}+x^{3}+y\right)\right]^{m}
$$

and

$$
\begin{aligned}
T\left(G_{2,2, e_{g}, m ; c}, x, y\right) & =\frac{1}{x-1}\left[\left\{x^{e_{g}}\left(x+x^{2}+x^{3}+y\right)\right\}^{m}\right. \\
& \left.+(x y-x-y)(2 x+y+1)^{m}\right] .
\end{aligned}
$$

Various evaluations of $T\left(G_{e_{1}, e_{2}, e_{g}, m ; o}, x, y\right)$ and $T\left(G_{e_{1}, e_{2}, e_{g}, m ; c}, x, y\right)$ for special values of the arguments $x$ and $y$ are of interest. In particular, for $x=1$, we have

$$
T\left(G_{e_{1}, e_{2}, e_{g}, m ; o}, 1, y\right)=(p+y-1)^{m}
$$

and

$$
T\left(G_{e_{1}, e_{2}, e_{g}, m ; c}, 1, y\right)=(p+y-1)^{m-1}\left[\left(m e_{g}+y-1\right)(p+y-1)\right.
$$




$$
\left.+\frac{m}{2}\left\{p(p-1)-e_{1}\left(e_{1}-1\right)-e_{2}\left(e_{2}-1\right)\right\}\right] \text {. }
$$

\section{Potts Model Partition Functions}

The equivalent Potts model partition functions are

$$
Z\left(G_{e_{1}, e_{2}, e_{g}, m ; o}, q, v\right)=q\left(\lambda_{Z, 0}\right)^{m}
$$

and

$$
Z\left(G_{e_{1}, e_{2}, e_{g}, m ; c}, q, v\right)=\left(\lambda_{Z, 0}\right)^{m}+(q-1)\left(\lambda_{Z, 1}\right)^{m}
$$

where

$$
\lambda_{Z, 0}=Z_{r}\left(T_{e_{g}+1}, q, v\right) Z_{r}\left(C_{p}, q, v\right)=(q+v)^{e_{g}} q^{-1}\left[(q+v)^{p}+(q-1) v^{p}\right]
$$

and

$$
\begin{aligned}
& \lambda_{Z, 1}=v^{e_{g}}\left[v^{e_{2}} Z_{r}\left(C_{e_{1}}, q, v\right)+v^{e_{1}} Z_{r}\left(C_{e_{2}}, q, v\right)-v^{p}\right] \\
= & v^{e_{g}}\left[q^{-1}\left[v^{e_{2}}\left\{(q+v)^{e_{1}}+(q-1) v^{e_{1}}\right\}+v^{e_{1}}\left\{(q+v)^{e_{2}}+(q-1) v^{e_{2}}\right\}\right]-v^{p}\right] .
\end{aligned}
$$

We note that

$$
\lambda_{Z, 0}=\lambda_{Z, 1}=v^{p+e_{g}-1}(p+v) \quad \text { at } q=0
$$

and

$$
\lambda_{Z, 1}=v^{e_{g}+1}\left[2(v+1)-v^{p-1}\right] \quad \text { for } \quad e_{1}=e_{2}=1
$$

\section{Chromatic Polynomials}

For the special case $v=-1$, our results for $Z\left(G_{e_{1}, e_{2}, e_{g}, m ; o}, q, v\right)$ and $Z\left(G_{e_{1}, e_{2}, e_{g}, m ; c}, q, v\right)$ reduce to to the chromatic polynomials given (for $e_{1} \geq 1$ and $e_{2} \geq 1$ ) in [1],

$$
P\left(G_{e_{1}, e_{2}, e_{g}, m ; o}, q\right)=q\left(\lambda_{P, 0}\right)^{m}
$$

and

$$
P\left(G_{e_{1}, e_{2}, e_{g}, m ; c}, q\right)=\left(\lambda_{P, 0}\right)^{m}+(q-1)\left(\lambda_{P, 1}\right)^{m}
$$

where

$$
\lambda_{P, 0}=(q-1)^{e_{g}+1} D_{p}
$$

and

$$
\lambda_{P, 1}=(-1)^{p+e_{g}} q^{-1}\left[(1-q)^{e_{1}}+(1-q)^{e_{2}}+q-2\right]
$$




$$
=(-1)^{p+e_{g}}\left[1-p-\sum_{s=2}^{e_{1}}\left(\begin{array}{c}
e_{1} \\
s
\end{array}\right)(-q)^{s-1}-\sum_{s=2}^{e_{2}}\left(\begin{array}{c}
e_{2} \\
s
\end{array}\right)(-q)^{s-1}\right] .
$$

(If either $e_{1}=0$ or $e_{2}=0$, the graphs contain one or more loops and the chromatic polynomial vanishes identically.)

\section{Free Energy}

It is of interest to remark on some thermodynamic properties of the $q$-state Potts model on the infinite-length limit of these polygon chain graphs. Although our results for the partition function apply for general $q$, we shall restrict our attention here to integer $q \geq 2$. We denote the $m \rightarrow \infty$ limit of the chain graph $G_{e_{1}, e_{2}, e_{g}, m, B C}$ as $\left\{G_{e_{1}, e_{2}, e_{g} ; B C}\right\}$. The reduced, dimensionless free energy per vertex for the Potts model in this limit is independent of boundary conditions, so we drop the $B C$ subscript. We find

$$
\begin{aligned}
f\left(\left\{G_{e_{1}, e_{2}, e_{g}}\right\}, q, v\right) & =\lim _{m \rightarrow \infty} \frac{1}{n} \ln \left[Z\left(G_{e_{1}, e_{2}, e_{g}, m ; B C}\right)\right] \\
& =\frac{1}{\left(p+e_{g}-1\right)} \ln \left(\lambda_{Z 0}\right) \\
& =\frac{1}{\left(p+e_{g}-1\right)} \ln \left[(q+v)^{e_{g}}\left\{(q+v)^{p}+(q-1) v^{p}\right\}\right] .
\end{aligned}
$$

(The actual Gibbs free energy per vertex is $\mathcal{G}=-k_{B} T f$.) As was discussed in [4], if one were to start with a Potts model partition function $Z(G, q, v)$ for a cyclic strip graph $G$ with variable real (positive) $q$, then, for a set of special (s) values of $q$, denoted $\left\{q_{s}\right\}$, one could encounter the noncommutativity

$$
\lim _{n \rightarrow \infty} \lim _{q \rightarrow q_{s}} Z(G, q, v)^{1 / n} \neq \lim _{q \rightarrow q_{s}} \lim _{n \rightarrow \infty} Z(G, q, v)^{1 / n} .
$$

For $G_{e_{1}, e_{2}, e_{g}, m ; c},\left\{q_{s}\right\}=\{0,1\}$. Since we are only interested in (integral) $q \geq 2$ here, we do not encounter any such noncommutativity.

Because these chain graphs are quasi-one-dimensional, the Potts model (with either sign of $J$ ) does not exhibit any critical behavior at nonzero temperature. However, it is of interest to investigate how thermodynamic quantities depend on the graphical parameters $e_{1}, e_{2}$, and $e_{g}$. A first observation is that the reduced free energy $f$ and hence quantities that are obtained as derivatives of $f$ with respect to temperature $T$, such as the internal energy per site, $U=-\partial f / \partial \beta$ and specific heat per site, $C=d U / d T$, only depend on $e_{1}$ and $e_{2}$ through their sum, $p$. This property follows from the fact that (in the limit $m \rightarrow \infty) f$ is determined completely by the dominant $\lambda$ (i.e., the $\lambda$ with the largest magnitude), namely $\lambda_{Z, 0}$, and this only depends on $e_{1}$ and $e_{2}$ via their sum, p. As an explicit example, the internal energy per site is

$$
U=-\frac{J(v+1)}{\left(p+e_{g}-1\right)}\left[\frac{e_{g}}{q+v}+p\left[\frac{(q+v)^{p-1}+(q-1) v^{p-1}}{(q+v)^{p}+(q-1) v^{p}}\right]\right] .
$$


In comparison, we recall that for the Potts model on the line, $U_{1 D}=-J(v+1) /(q+v)$. In the expression (6.3) for $U$, one thus sees the interplay of the underlying circuit graph including the $m$ line segments, each of $e_{g}$ edges, with the $m p$-sided polygons, in the limit as $m \rightarrow \infty$. The limit of Eq. (6.3) as the temperature $T \rightarrow \infty$, i.e., $v \rightarrow 0$, is

$$
U=-\frac{J}{q}\left(\frac{p+e_{g}}{p+e_{g}-1}\right) \text { at } v=0 .
$$

Since $\left(p+e_{g}\right) /\left(p+e_{g}-1\right)>1, U(v=0)$ is more negative than the analogous infinitetemperature limit of $U$ for the Potts model on the line, namely $U(0)_{1 D}=-J / q$. One can also consider the zero-temperature limit. For the antiferromagnet, this reverts back to the analysis of the chromatic polynomial, as in [1]. For the ferromagnet, as $\beta \rightarrow \infty$,

$$
\lim _{\beta \rightarrow \infty} U=-J\left(\frac{p+e_{g}}{p+e_{g}-1}\right) \text { for } J>0 .
$$

Again, this is more negative than the corresponding expression for the Potts ferromagnet on the line, which is $\lim _{\beta \rightarrow \infty} U_{1 D}=-J$. In both the high- and low-temperature limits, these differences can be attributed to the additional spin-spin interactions due to the combination of the repeated polygons attached to the underlying global circuit graph.

Finally, we note some limiting cases:

$$
\lim _{e_{g} \rightarrow \infty} f\left(\left\{G_{e_{1}, e_{2}, e_{g}}\right\}, q, v\right)=\ln (q+v) \quad \text { for fixed finite } p
$$

and

$$
\lim _{p \rightarrow \infty} f\left(\left\{G_{e_{1}, e_{2}, e_{g}}\right\}, q, v\right)=\ln (q+v) \text { for fixed finite } e_{g} .
$$

In both of these cases, the dimensionless free energy per site thus reduces to that of the $n \rightarrow \infty$ limit of the Potts model on a line, $f=\ln (q+v)$. This reduction can be ascribed to the dominance of one of the two structural parts of the graph - the global circuit in Eq. (6.6) and the polygons in Eq. (6.7).

\section{Zeros of the Partition Function and Locus $\mathcal{B}$}

It is also of interest to consider the locus of zeros of the partition function. In the limit $m \rightarrow \infty$, some zeros may merge to form curves denoted as the locus $\mathcal{B}$. It is convenient to consider this locus in the $q$ plane for fixed temperature variable $v$. For the case $v=-1$, i.e., the $T=0$ Potts antiferromagnet, $\mathcal{B}$ was studied in [1]. Here we can use our general results to analyze the case of the Potts antiferromagnet at temperatures above $T=0$ and the Potts ferromagnet. Thus, we consider $\mathcal{B}$ for $v$ in the full physical range, $v \geq-1$.

For the open polygon chain graph, since $Z\left(G_{e_{1}, e_{2}, e_{g}, m ; o}, q, v\right)$ involves just the $m$ 'th power of a single $\lambda$ term, it has a fixed set of discrete zeros, independent of $m$, with no continuous locus, i.e., $\mathcal{B}=\emptyset$. For the $m \rightarrow \infty$ limit of the cyclic polygon chain graph, $\mathcal{B}$ is a nontrivial locus, determined by the equation $\left|\lambda_{Z, 0}\right|=\left|\lambda_{Z, 1}\right|$. For this limit, with $v=-1$, several properties of $\mathcal{B}$ were derived in [1], namely, that $\mathcal{B}$ (i) is compact, (ii) passes through $q=0$, and (iii) encloses regions in the $q$ plane. With the notation 
$e_{s}$ and $e_{\ell}$ denoting the smaller and larger of $e_{1}$ and $e_{2}$, it was shown in [1] that (iv) if $e_{s}=1$, then $\mathcal{B}$ is the circle $|q-1|=1$, independent of the values of $e_{\ell}$ and $e_{g}$, so that $q_{c}=2$, where $q_{c}$ is defined as the maximal point at which $\mathcal{B}$ crosses the real axis (which it always does for the $m \rightarrow \infty$ limit of these cyclic polygon chain graphs). Two additional properties concerning $q_{c}$ values for $v=-1$ (denoted (B5) and (B6) in [1]) were also shown.

We use the notation $\lim _{m \rightarrow \infty} G_{e_{1}, e_{2}, e_{g}, m ; c} \equiv\left\{G_{e_{1}, e_{2}, e_{g} ; c}\right\}$. For this $m \rightarrow \infty$ limit of the cyclic polygon chain graph, we find that properties (i)-(iii) continue to hold. The proof of the compactness property (i) is a generalization of the proof for $v=-1$ given in [1]; one uses the fact that a necessary and sufficient condition that $\mathcal{B}$ is noncompact in the $q$ plane, passing through $1 / q=0$, is that the equation $\left|\lambda_{Z, 0}\right|=\left|\lambda_{Z, 1}\right|$ has a solution for $1 / q=0$. To show that this is not the case, we extract a factor of $q^{p+e_{g}-1}$ from both sides of this equation and define $\lambda_{Z, d} \equiv q^{p+e_{g}-1} \bar{\lambda}_{Z, d}$ for $d=0,1$. Dividing both sides by the factor $\left|q^{p+e_{g}-1}\right|$ we have $\left|\bar{\lambda}_{Z, 0}\right|=\left|\bar{\lambda}_{Z, 1}\right|$. Taking $1 / q \rightarrow 0$, we see that this equation cannot be satisfied because $\bar{\lambda}_{Z, 0} \rightarrow 1$ while $\bar{\lambda}_{Z, 1} \rightarrow 0$. This proves the compactness of $\mathcal{B}$ for general $v$. Property (ii) follows because, as Eq. (4.5) shows, $\left|\lambda_{Z, 0}\right|=\left|\lambda_{Z, 1}\right|$ at $q=0$. To show property (iii), we evaluate $f$ in Eq. (6.1) (away from $q_{s}$ values so as to avoid the noncommutativity). The result is that in region $R_{1}$, which contains the semi-infinite real intervals $q>q_{c}$ and $q<0, \lambda_{Z, 0}$ has a larger magnitude than $\lambda_{Z, 1}$, so $f=\left(p+e_{g}-1\right)^{-1} \ln \lambda_{Z, 0}$, as in Eq. (6.1), while in a region $R_{2}$ containing an interval of small positive $q$ neighboring the origin, $\lambda_{Z, 1}$ has a larger magnitude than $\lambda_{Z, 0}$, so $f=\left(p+e_{g}-1\right)^{-1} \ln \lambda_{Z, 1}$. If these regions were not completely separated by the nonanalytic boundary $\mathcal{B}$, then one could analytically continue $f$ from one to the other, but this would lead to a contradiction, since $f$ has a different functional form in these two regions. This proves that $\mathcal{B}$ separates the $q$ plane into regions. Property (iv) for $v=-1$ was a special consequence of the complete intersection theorem for chromatic polynomials, namely that if a graph $G$ is the union $G=G_{1} \cup G_{2}$ such that the intersection $G_{1} \cap G_{2}=K_{r}$, where $K_{r}$ is the complete graph on $r$ vertices (i.e, the graph in which each vertex is connected to every other vertex by an edge), then $P(G, q)=P\left(G_{1}, q\right) P\left(G_{2}, q\right) / P\left(K_{r}, q\right)$, where $P\left(K_{r}, q\right)=\prod_{s=0}^{r-1}(q-s)$. This theorem does not apply for $v \neq-1$, so the property (iv) for $v=-1$ no longer holds for $v>-1$.

We have obtained a number of results on $q_{c}$ for the general case $v>-1$. As we have discussed above, for $\left\{G_{e_{1}, e_{2}, e_{g} ; c}\right\}$, the locus $\mathcal{B}$ crosses the real $q$ axis at $q=0$ and at a maximal point denoted $q_{c}$. (Depending on the values of $e_{1}, e_{2}, e_{g}$, and $v, \mathcal{B}$ may cross the $q$ axis at additional points besides $q=0$ and $q_{c}$.) Some exact results on $q_{c}$ are

$$
\begin{aligned}
& q_{c}\left(\left\{G_{1,1,0 ; c}\right\}\right)=-2 v(v+2) \\
& q_{c}\left(\left\{G_{1,1,1 ; c}\right\}\right)=-v(v+3)
\end{aligned}
$$

and

$$
q_{c}\left(\left\{G_{2,2, e_{g} ; c}\right\}\right)=-2 v \quad \forall e_{g} .
$$


For most cases, $q_{c}$ is a root of a higher-order equation. For example, for $\left\{G_{1,1,2 ; c}\right\}, q_{c}$ is the maximal real root of the cubic equation

$$
q^{3}+v(v+4) q^{2}+v^{2}(2 v+5) q+2 v^{3}(v+2)=0 .
$$

These calculations may be compared with our previous results for $q_{c}$ for the infinitelength limits of some other strips with periodic longitudinal boundary conditions. Denoting $\lim _{n \rightarrow \infty} C_{n}$ as $\{C\}$, one has $q_{c}(\{C\})=-2 v$, the same as $q_{c}\left(\left\{G_{2,2, e_{g} ; c}\right\}\right)$. For the cyclic or Möbius square-lattice ladder strip [4],

$$
q_{c}(\{s q l a d\})=-v(v+3),
$$

the same as $q_{c}\left(\left\{G_{1,1,1 ; c}\right\}\right)$. For the cyclic or Möbius ladder strip of the triangular lattice [11], and also the self-dual (sd) square-lattice ladder strip,

$$
q_{c}(\{\text { tri lad }\})=q_{c}\left(\left\{s q_{s d} l a d\right\}\right)=-\frac{v(2 v+5)}{v+2} .
$$

These results for $q_{c}(\{G\})$ have the general property that, as $v$ increases from -1 to 0 , i.e., as the temperature increases from 0 to infinity for the Potts antiferromagnet, they decrease monotonically from their $v=-1$ values to 0 , and in the ferromagnetic region, $v \geq 0$, they are negative. One may also consider the accumulation locus of partition function zeros in the $v$ plane for fixed $q$. However, this is of somewhat less interest than $\mathcal{B}$ in the $q$ plane for fixed $v$, since these polygon chain graphs are quasi-one-dimensional and hence (for $q \geq 2$ ) $\mathcal{B}$ does not cross the real $v$ axis at any point corresponding to nonzero temperature.

\section{Flow Polynomials}

An interesting problem in graph theory is the task of enumerating discretized flows on the edges of a (connected) $G$ that satisfy flow conservation at each vertex, i.e. for which there are no sources or sinks. The flow on each edge can take on any of $q$ values modulo $q$, $(\operatorname{so} q=0 \bmod q)$. One arbitrarily chooses a direction for each edge of $G$ and assigns a discretized flow value to it. The value zero is excluded, since it is equivalent to the edge being absent from $G$; henceforth, we take a $q$-flow to mean implicitly a nowhere-zero $q$-flow. The flow or current conservation condition is that the flows into any vertex must be equal, $\bmod q$, to the flows outward from this vertex. These are called $q$-flows on $G$, and the number of these is given by the flow polynomial $F(G, q)$. This is a special case of the Tutte polynomial for $x=0$ and $y=1-q$ :

$$
F(G, q)=(-1)^{c(G)} T(G, 0,1-q) .
$$

A bridge on a graph $G$ is defined as an edge with the property that if it is deleted, this increases the number of connected components of $G$ by one. If a (connected) graph $G$ contains any bridge, then the flow polynomial vanishes identically. The open strip graph $G_{e_{1}, e_{2}, e_{g}, m ; o}$ contains at least one bridge if $e_{g} \geq 1$, and therefore it does not allow any $q$-flows:

$$
F\left(G_{e_{1}, e_{2}, e_{g}, m ; o}, q\right)=0 \text { if } e_{g} \geq 1 .
$$


For $e_{g}=0$, we find

$$
F\left(G_{e_{1}, e_{2}, e_{g}, m ; o}, q\right)=(q-1)^{m} \text { if } e_{g}=0 .
$$

Note that this is equal to $\left[F\left(C_{p}, q\right)\right]^{m}$, showing that the flows occur independently in each $p$-gon circuit graph. Algebraically, this follows from the factorized form of $T\left(G_{e_{1}, e_{2}, e_{g}, m ; o}, x, y\right)$ in Eq. (3.1). This is also clear since, if one considers the flows in the $p$-gons forming the ends of the open chain, these have nowhere else to flow, and, since that is the case, the same is true for the flows in all of the $p$-gons that form the interior of the chain.

Using our calculation of the Tutte polynomial of $G_{e_{1}, e_{2}, e_{g}, m ; c}$, we find, for the cyclic strip,

$$
F\left(G_{e_{1}, e_{2}, e_{g}, m ; c}, q\right)=\left\{\begin{array}{ll}
(q-1)(q-2)^{m} & \text { if } e_{g} \geq 1 \\
(q-1)(q-2)^{m}+(q-1)^{m} & \text { if } e_{g}=0
\end{array} .\right.
$$

Thus, both for $e_{g}=0$ and for $e_{g} \geq 1$, the cyclic polygon chain graph allows more $q$ flows than the open polygon chain graph. This follows because of the freedom of the flows in the cyclic case to make a global circuit around the chain. These calculations for the cyclic polygon chain may also be compared with the result $F\left(C_{n}, q\right)=q-1$. One sees that if $e_{g}=0$, then there are more flows on the $G_{e_{1}, e_{2}, e_{g}, m ; c}$ graphs, owing to the possibility of flows within each polygon. However, if $q=2$ and if $e_{g} \geq 1$, then, owing to the degree-3 vertices where the polygons connect onto the line segments in the chain, no $q$-flows can occur, since the flow conservation condition cannot be satisfied at these vertices.

Since these results hold for arbitrary chain length $m$, one may consider the limit of infinite length and define, as in Ref. [12, a function $\phi$ representing the number of $q$-flows per face of $G$ in this limit,

$$
\phi\left(\left\{G_{e_{1}, e_{2}, e_{g} ; B C}, q\right)=\lim _{m \rightarrow \infty}\left[F\left(G_{e_{1}, e_{2}, e_{g}, m ; B C}, q\right)\right]^{1 / f c(G)},\right.
$$

where $f c(G)$ denotes the number of faces of $G$. Here, $f c\left(G_{e_{1}, e_{2}, e_{g}, m ; o}\right)=m+1$ and $f c\left(G_{e_{1}, e_{2}, e_{g}, m ; c}\right)=m+2$. Unlike the free energy, the function $\phi$ does depend on whether one uses open or cyclic boundary conditions, since some flows make global circuits around the chain in the cyclic case. For $q \geq 2$ (so that nowhere-zero $q$-flows can occur), we calculate

$$
\phi\left(\left\{G_{e_{1}, e_{2}, e_{g} ; o}\right\}, q\right)= \begin{cases}0 & \text { if } e_{g} \geq 1 \\ q-1 & \text { if } e_{g}=0\end{cases}
$$

and

$$
\phi\left(\left\{G_{e_{1}, e_{2}, e_{g} ; c}\right\}, q\right)=\left\{\begin{array}{ll}
q-2 & \text { if } e_{g} \geq 1 \\
q-1 & \text { if } e_{g}=0
\end{array} .\right.
$$




\section{Reliability Polynomials}

A communication network, such as the internet, can be represented by a graph, with the vertices of the graph representing the nodes of the network and the edges of the graph representing the communication links between these nodes. In analyzing the reliability of a network, one is interested in the probability that there is a working communications route between any node and any other node. This is called the all-terminal reliability function. This is commonly modeled by a simplification in which one assumes that each node is operating with probability $p_{\text {node }}$ and each link (abbreviated $\ell$ ) is operating with probability $p_{\ell}$. As probabilities, $p_{\text {node }}$ and $p_{\ell}$ lie in the interval $[0,1]$. The dependence of the all-terminal reliability function $R_{\text {tot }}\left(G, p_{\text {node }}, p_{\ell}\right)$ on $p_{\text {node }}$ is an overall factor of $\left(p_{\text {node }}\right)^{n}$; i.e., $R_{\text {tot }}\left(G, p_{\text {node }}, p_{\ell}\right)=\left(p_{\text {node }}\right)^{n} R\left(G, p_{\ell}\right)$. The difficult part of the calculation of $R_{\text {tot }}\left(G, p_{\text {node }}, p_{\ell}\right)$ is thus the part that depends on the links, $R\left(G, p_{\ell}\right)$. The function $R\left(G, p_{\ell}\right)$ is given by

$$
R\left(G, p_{\ell}\right)=\sum_{\tilde{G} \subseteq G} p_{\ell}^{e(\tilde{G})}\left(1-p_{\ell}\right)^{e(G)-e(\tilde{G})}
$$

where $\tilde{G}$ is a connected spanning subgraph of $G$. Each term in this sum is the probability that the communication links $\tilde{E} \in \tilde{G}$ are functioning (equal to $p_{\ell}^{e(\tilde{G})}$ ) times the probability that the other links, $E-\tilde{E}$, are not functioning (equal to $\left(1-p_{\ell}\right)^{e(G)-e(\tilde{G})}$ ). From its definition, $R\left(G, p_{\ell}\right)$ is clearly a monotonically increasing function of $p_{\ell} \in[0,1]$ with the boundary values $R(G, 0)=0$ and $R(G, 1)=1 . R\left(G, p_{\ell}\right)$ is given in terms of the Tutte polynomial, evaluated with $x=1$ (guaranteeing that $\tilde{G}$ is a connected spanning subgraph of $G$ ) and $y=y_{\ell}$, where

$$
y_{\ell}=\frac{1}{1-p_{\ell}} \quad \text { i.e., } v_{\ell}=y_{\ell}-1=\frac{p_{\ell}}{1-p_{\ell}},
$$

by the relation

$$
R\left(G, p_{\ell}\right)=p_{\ell}^{n-1}\left(1-p_{\ell}\right)^{\ell(G)+1-n} T\left(G, 1, \frac{1}{1-p_{\ell}}\right) .
$$

Using our calculation of the Tutte polynomials for $G_{e_{1}, e_{2}, e_{g}, m ; o}$ and $G_{e_{1}, e_{2}, e_{g}, m ; c}$, we find

$$
R\left(G_{e_{1}, e_{2}, e_{g}, m ; o}, p_{\ell}\right)=\left[p_{\ell}^{p+e_{g}-1}\left\{p\left(1-p_{\ell}\right)+p_{\ell}\right\}\right]^{m}
$$

and

$$
\begin{aligned}
& R\left(G_{e_{1}, e_{2}, e_{g}, m ; c}, p_{\ell}\right)=p_{\ell}^{\left(p+e_{g}-1\right) m}\left[p\left(1-p_{\ell}\right)+p_{\ell}\right]^{m-1} \times \\
\times & {\left[\left\{m e_{g}\left(1-p_{\ell}\right)+p_{\ell}\right\}\left\{p\left(1-p_{\ell}\right)+p_{\ell}\right\}\right.} \\
+ & \left.\frac{m}{2}\left(1-p_{\ell}\right)^{2}\left\{p(p-1)-e_{1}\left(e_{1}-1\right)-e_{2}\left(e_{2}-1\right)\right\}\right] .
\end{aligned}
$$

In general, $R\left(G_{e_{1}, e_{2}, e_{g}, m ; c}, p_{\ell}\right) \geq R\left(G_{e_{1}, e_{2}, e_{g}, m ; o}, p_{\ell}\right)$, with equality only at $p_{\ell}=0,1$. This can be understood as a consequence of the fact that with the cyclic boundary condition, 
there are more possible communication routes linking two nodes than there are with the open boundary condition. We observe that for $p_{\ell} \in(0,1), R\left(G_{e_{1}, e_{2}, e_{g}, m ; o}, p_{\ell}\right)$ and $R\left(G_{e_{1}, e_{2}, e_{g}, m ; c}, p_{\ell}\right)$ are (i) decreasing functions of $m$ for fixed $e_{1}, e_{2}$, and $e_{g}$; (ii) decreasing functions of $e_{g}$ for fixed $e_{1}, e_{2}$, and $m$; and (iii) decreasing functions of $e_{1}$ for fixed $e_{2}, e_{g}$, and $m$. These properties can be ascribed to the greater probability of communication bottlenecks as the respective parameter, $m, e_{g}$, or $e_{1}$ increases with the other parameters held fixed. We also observe that for fixed $p=e_{1}+e_{2}, e_{g}$, and $m$, these reliability polynomials increase as $\left|e_{1}-e_{2}\right|$ decreases.

As in [13], in the limit of infinite chain length, $m \rightarrow \infty$, one may define a function $r$ that measures the reliability per node, as

$$
r\left(\left\{G_{e_{1}, e_{2}, e_{g}}\right\}, p_{\ell}\right)=\lim _{m \rightarrow \infty}\left[R\left(G_{e_{1}, e_{2}, e_{g}, m ; B C}, p_{\ell}\right)\right]^{1 / n} .
$$

As was discussed in [13] for other strip graphs, this function is independent of the longitudinal boundary conditions, so we drop the $B C$ subscript on the left-hand side of Eq. (9.6). Clearly, for a general $\{G\}, r\left(\{G\}, p_{\ell}\right)$ is an increasing function of $p_{\ell} \in[0,1]$ with the values $r(\{G\}, 0)=0$ and $r(\{G\}, 1)=1$. From our exact calculations above, we find

$$
r\left(\left\{G_{e_{1}, e_{2}, e_{g}}\right\}, p_{\ell}\right)=p_{\ell}\left[p\left(1-p_{\ell}\right)+p_{\ell}\right]^{\frac{1}{p+e_{g}-1}} .
$$

As a comparison, for the infinite-length limit of a line graph $L_{n}$ or circuit graph $C_{n}$, $r\left(\{\mathcal{L}\}, p_{\ell}\right)=r\left(\{\mathcal{C}\}, p_{\ell}\right)=p_{\ell} \equiv r_{1 D}\left(p_{\ell}\right)$. Now for $p_{\ell} \in(0,1)$ (and $p \geq 2$ ), the factor $\left[p\left(1-p_{\ell}\right)+p_{\ell}\right]^{1 /\left(p+e_{g}-1\right)}>1$, and hence $r\left(\left\{G_{e_{1}, e_{2}, e_{g}}\right\}, p_{\ell}\right)>r_{1 D}\left(p_{\ell}\right)$. Concerning the dependence on $e_{1}$ and $e_{2}$ (which only enter in the form of their sum, $p$ ) and on $e_{g}$, we find from an analysis of the respective partial derivatives, for a fixed $p_{\ell} \in(0,1)$, that $r\left(\left\{G_{e_{1}, e_{2}, e_{g}}\right\}, p_{\ell}\right)$ is a monotonically decreasing function (i) of $p$, for fixed $e_{g}$, and (ii) of $e_{g}$, for fixed $p$. Furthermore,

$$
\lim _{p \rightarrow \infty} r\left(\left\{G_{e_{1}, e_{2}, e_{g}}\right\}, p_{\ell}\right)=p_{\ell}
$$

and

$$
\lim _{e_{g} \rightarrow \infty} r\left(\left\{G_{e_{1}, e_{2}, e_{g}}\right\}, p_{\ell}\right)=p_{\ell} .
$$

Thus, in both of these limits, $r\left(\left\{G_{e_{1}, e_{2}, e_{g}}\right\}, p_{\ell}\right)$ reduces to $r_{1 D}\left(p_{\ell}\right)$.

\section{Percolation Clusters}

In this section we use our results to calculate a quantity of interest in the area of bond percolation. We first briefly mention some necessary background. Consider a connected graph $G$ and assume that the vertices are definitely present, but each edge is present only with a probability $p_{\ell} \in[0,1]$. In the usual statistical mechanical context, one usually considers a limit in which the number of vertices $n \rightarrow \infty$. An important quantity is the average number of connected components (= clusters) in $G$. For a given $G$, we denote 
this average cluster number per vertex as $\langle k\rangle_{G}$. This is given by

$$
\begin{aligned}
\langle k\rangle_{G} & =\frac{(1 / n) \sum_{G^{\prime}} k\left(G^{\prime}\right) p_{\ell}^{e\left(G^{\prime}\right)}\left(1-p_{\ell}\right)^{e(G)-e\left(G^{\prime}\right)}}{\sum_{G^{\prime}} p_{\ell}^{e\left(G^{\prime}\right)}\left(1-p_{\ell}\right)^{e(G)-e\left(G^{\prime}\right)}} \\
& =\frac{(1 / n) \sum_{G^{\prime}} k\left(G^{\prime}\right) v_{\ell}^{e\left(G^{\prime}\right)}}{\sum_{G^{\prime}} v_{\ell}^{e\left(G^{\prime}\right)}},
\end{aligned}
$$

where $G^{\prime}$ is a spanning subgraph of $G$, as above, and $v_{\ell}$ was defined in Eq. (9.2). Hence, in the $n \rightarrow \infty$ limit, the average cluster number per vertex, $\langle k\rangle_{\{G\}}$, is given by

$$
\langle k\rangle_{\{G\}}=\left.\frac{\partial f(\{G\}, q, v)}{\partial q}\right|_{q=1, v=v_{\ell}} .
$$

Using Eq. (10.2) with Eq. (6.1), we find, for the infinite-length limits of both the open and cyclic polygon chains, the average cluster number

$$
\begin{aligned}
\langle k\rangle_{\left\{G_{\left.e_{1}, e_{2}, e_{g}\right\}}\right.} & =\frac{1}{\left(p+e_{g}-1\right)}\left[\left(p+e_{g}\right)\left(1-p_{\ell}\right)+p_{\ell}^{p}-1\right] \\
& =\left(\frac{1-p_{\ell}}{p+e_{g}-1}\right)\left[p+e_{g}-\sum_{j=0}^{p-1} p_{\ell}^{j}\right] .
\end{aligned}
$$

This may be compared with the result $\langle k\rangle_{1 D}=1-p_{\ell}$ for the infinite line. We have

$$
\begin{aligned}
\langle k\rangle_{1 D}-\langle k\rangle_{\left\{G_{e_{1}, e_{2}, e_{g}}\right\}} & =\left(\frac{1-p_{\ell}}{p+e_{g}-1}\right)\left[\left(\sum_{j=0}^{p-1} p_{\ell}^{j}\right)-1\right] \\
& \geq 0
\end{aligned}
$$

with equality holding only if $p_{\ell}=1$ or $p=1$. Thus, if $p_{\ell}<1$ and $p \geq 2$, the average number of clusters per vertex is greater for the line than for the infinite-length limit of the polygon chain graph (with either set of boundary conditions).

\section{Some Graphical Quantities}

Special valuations of the Tutte polynomial of a graph yield various quantities describing properties of this graph. In this section we give these. First, we recall some definitions. A tree graph is a connected graph with no circuits. A spanning tree of a graph $G$ is a spanning subgraph of $G$ that is also a tree. A spanning forest of a graph $G$ is a spanning subgraph of $G$ that may consist of more than one connected component but contains no circuits. The special valuations of interest here are (i) $T(G, 1,1)=N_{S T}(G)$, the number of spanning trees $(S T)$ of $G$; (ii) $T(G, 2,1)=N_{S F}(G)$ the number of spanning forests $(S F)$ of $G$; (iii) $T(G, 1,2)=N_{C S S G}(G)$, the number of connected spanning subgraphs $(C S S G)$ of $G$; and (iv) $T(G, 2,2)=N_{S S G}(G)=2^{e(G)}$, the number of spanning subgraphs $(S S G)$ of $G$. For both the open and cyclic strips, the last of these quantities is directly determined by Eq. (1.4) (without the necessity of calculating the Tutte polynomial) to be

$$
N_{S S G}\left(G_{e_{1}, e_{2}, e_{g}, m ; o}\right)=N_{S S G}\left(G_{e_{1}, e_{2}, e_{g}, m ; c}\right)=2^{\left(p+e_{g}\right) m} .
$$


We evaluate our general results for $T\left(G_{e_{1}, e_{2}, e_{g}, m ; o}, x, y\right)$ and $T\left(G_{e_{1}, e_{2}, e_{g}, m ; c}, x, y\right)$ to obtain the quantities (i)-(iii). For the numbers of spanning trees, we find

$$
N_{S T}\left(G_{e_{1}, e_{2}, e_{g}, m ; o}\right)=p^{m}
$$

and

$$
N_{S T}\left(G_{e_{1}, e_{2}, e_{g}, m ; c}\right)=m p^{m-1}\left[p e_{g}+\frac{1}{2}\left\{p(p-1)-e_{1}\left(e_{1}-1\right)-e_{2}\left(e_{2}-1\right)\right\}\right] .
$$

The numbers of spanning forests are

$$
N_{S F}\left(G_{e_{1}, e_{2}, e_{g}, m ; o}\right)=\left[2^{e_{g}}\left(2^{p}-1\right)\right]^{m}
$$

and

$$
N_{S F}\left(G_{e_{1}, e_{2}, e_{g}, m ; c}\right)=\left[2^{e_{g}}\left(2^{p}-1\right)\right]^{m}-\left[2^{e_{1}}+2^{e_{2}}-2\right]^{m} .
$$

The numbers of connected spanning subgraphs are

$$
N_{C S S G}\left(G_{e_{1}, e_{2}, e_{g}, m ; o}\right)=(p+1)^{m}
$$

and

$$
\begin{aligned}
N_{C S S G}\left(G_{e_{1}, e_{2}, e_{g}, m ; c}\right) & =(p+1)^{m-1}\left[\left(m e_{g}+1\right)(p+1)\right. \\
& \left.+\frac{m}{2}\left\{p(p-1)-e_{1}\left(e_{1}-1\right)-e_{2}\left(e_{2}-1\right)\right\}\right] .
\end{aligned}
$$

We also give another evaluation of the Tutte polynomial. For a general connected graph $G=(E, V)$, one can define an orientation of of $G$, i.e., a directed graph $\vec{G}=(V, \vec{E})$ by assigning a direction to each edge $e \in E$. There are $2^{e(G)}$ of these orientations. Among these, an acyclic orientation of $G$ is defined as an orientation that does not contain any directed cycles. Here, a directed cycle is a cycle in which, as one travels along the cycle, all of the oriented edges have the same direction. The number of such acyclic orientations is denoted $a(G)$ and is given by the evaluation of the Tutte polynomial with $x=2$ and $y=0[14]$ :

$$
a(G)=T(G, 2,0) \text {. }
$$

Equivalently, this is obtained by the evaluation of the chromatic polynomial at $q=-1$ : $a(G)=(-1)^{n(G)} P(G,-1)$. From the results in [1], in agreement with our calculations here, we have, for the numbers of acyclic orientations of the open and cyclic polygon chain graphs

$$
a\left(G_{e_{1}, e_{2}, e_{g}, m ; o}\right)=\left[2^{e_{g}}\left(2^{p}-2\right)\right]^{m}
$$

and

$$
a\left(G_{e_{1}, e_{2}, e_{g}, m ; c}\right)=\left[2^{e_{g}}\left(2^{p}-2\right)\right]^{m}-2\left[2^{e_{1}}+2^{e_{2}}-3\right]^{m} .
$$




\section{Conclusions}

In conclusion, in this paper, generalizing our previous results on chromatic polynomials with S.-H. Tsai in [1], we have presented exact calculations of the Potts model partition functions and equivalent Tutte polynomials for a class of polygon chain graphs with open and cyclic boundary conditions. We have evaluated special cases of these results to compute the corresponding flow polynomials, reliability polynomials, and various quantities of graph-theoretic interest, and have analyzed the dependence on the parameters $e_{1}, e_{2}$, and $e_{g}$ characterizing the families of graphs.

\section{Acknowledgments}

This research was partially supported by the grant NSF-PHY-06-53342.

\section{References}

[1] Shrock R and Tsai S-H 1999 J. Phys. A 325053.

[2] Wu F Y 1982 Rev. Mod. Phys. 54235.

[3] Biggs N L, Damerell R M, and Sands D A 1972 J. Combin. Theory B 12123.

[4] Shrock R 2000 Physica A 283388.

[5] Chang S-C and Shrock R 2001 Physica A 296234.

[6] Chang S-C, Salas J, and Shrock R 2002 J. Stat. Phys. 1071207.

[7] Fortuin C M, Kasteleyn P W 1972 Physica 57536.

[8] Biggs N 1993 Algebraic Graph Theory (Cambridge Univ. Press, Cambridge, 2nd ed.).

[9] Bollobás B 1998 Modern Graph Theory (Springer, New York).

[10] Workshop on Zeros of Graph Polynomials 2008 Newton Institute for Mathematical Sciences, Cambridge University, http://www.newton.ac.uk/programmes/CSM/seminars.

[11] Chang S-C and Shrock R 2000 Physica A 286189.

[12] Chang S-C and Shrock R 2003 J. Stat. Phys. 112815.

[13] Chang S-C and Shrock R 2003 J. Stat. Phys. 1121019.

[14] Stanley R P 1973 Discrete Math. 5171. 